\title{
Theoretical and experimental study of energy storage process in metals under plastic deformation
}

\author{
by $\mathrm{A}$ by O. Plekhov, O.Naimark
}

Institute of continuous media mechanics of Russian Academy of Sciences, 1.ak. Korolev str., 614013 Perm,

Russia, poa@icmm.ru

\begin{abstract}
This work is devoted to the study of defect induced energy dissipation and storage processes in metals. Extending previous results of the research group in Perm, we proposed a statistical description of the mesodefect ensemble and thermodynamic internal variable model of heat dissipation in metals. The theoretical consideration is supported by experimental investigations of heat dissipation in submirocrystalline metals under fatigue loading and by investigation temperature evolution at fatigue crack tip.
\end{abstract}

\section{Introduction}

The evolution of microstructure in metallic materials has been the object of intensive studies during the all last century. It is well known that deformation process of metal, especially plastic flow is accompanied by high dislocation activity and the appearance of specific mesodefect patterns. The pattern evolution process accompanied by failure and rotation of mesovolumes of material leads to the creation of high internal stress fields and, as a consequence, to energy storage into the material. The ratio of the storage energy $\left(W_{s t}\right)$ to plastic work $\left(W_{p}=\tilde{\sigma}:\left(\widetilde{\varepsilon}-\widetilde{\varepsilon}^{e}\right)\right)$ is an issue during at list half of century [1-5]. This question was good reviewed in [6]. The generally accepted assumption $W_{s t}<0.2 W_{p}$ is often justified by citing the early pioneering work of G.I. Taylor [7] gives good results in mechanical calculations mainly at high strain rates [9]. At slow strain rates and in some particular cases such as, for instance, an application of infrared thermography for damage monitoring $[10,11]$ we need a adequate physical model of energy balance in a material.

This model should describe a simultaneously evolution of a number of non-linear interacting defects at different spatial scales. The description of these interactions requires both detailed theoretical investigation of the non-linear laws of defect kinetics and the development of new experimental techniques.

Recently a lot of experimental works discover interesting thermomechanical phenomena such as: thermal front propagation under quasi-static loading [12], Joule-Thomson effect in thermoelasticity [13], high temperature frequency generation under sinusoidal fatigue loading $[11,14]$ in solids and shows a lack in adequate theoretical models.

One of the first experimental investigations devoted to the measurement of the storage energy was made in [15]. Experimental [1] and thermodynamic $[16,17]$ consideration of the problem shows that the ratio $W_{s t} / W_{p}$ is historydependent quantity and adequate calculation of this value requests an introduction of some additional structural sensitive variables. Plastic deformation, often considered as such variable, cannot be interpreted as general thermodynamic variable.

One powerful way to introduce the structural sensitive variable and to obtain the nonlinear kinetic equations for the defect density based on the statistical physics approach was proposed in [18]. A solution of the statistical problem allows us to ascertain a specific non-linearity of the linear elastic solid free energy on the defect density tensor and to propose the phenomenology model of solid [19].

The present work is devoted to the theoretical and experimental study of heat dissipation process in metals based on the infrared thermography. In framework of this paper we illustrate the advantage of this technique by two experiments. We presented the results of self-heating (Risitano technique) test for submicrocrystalline metals and calculation of stress intensity factor under fatigue crack propagation.

The experimental results stimulate us to develop a thermodynamic internal variable model for defect evolution in metals. The model is based on the results of the statistical description of mesodefect evolution. The plastic deformation was divided into two parts and only one part (structural) is interpreted as thermo dynamical variables.

\section{Thermodynamic consideration}

Based on the data provided in works [14,20], a thermodynamic process can be described by a vector displacement field $\vec{u}(\vec{x}, t)$ and an absolute temperature field $T(\vec{x}, t)$. Here $\vec{x}$ is the position vector of a particle in a fixed reference configuration and $t$ is time. Thermodynamic process obeys the momentum balance equation and the first and second laws of thermodynamics. In the case of small deformations, these equations involve the following thermodynamic quantities: volumetric mass $\rho$, specific internal energy $e$, strain and stress tensors $\widetilde{\varepsilon}$ and $\tilde{\sigma}$, heat supply $r$, heat flux 
vector $\vec{q}$, specific Helmholtz free energy $F$, and specific entropy $\eta$. In particular, $\widetilde{\varepsilon}=\left(\nabla \vec{u}+\nabla \vec{u}^{T}\right) / 2$ and $\tilde{\sigma}$ is the Cauchy stress tensor. The energy balance and the entropy growth can be written as

$$
\begin{gathered}
\dot{e} \equiv(\dot{\mathcal{F}}+\eta \dot{T}+\dot{\eta} T)=\frac{1}{\rho} \tilde{\sigma}: \dot{\vec{\varepsilon}}+r-\bar{\nabla} \cdot \bar{q}, \\
\dot{\eta}-\bar{\nabla} \cdot\left(\frac{\bar{q}}{T}\right)-r^{\prime} \geq 0,
\end{gathered}
$$

where $\bar{\nabla}=\left(\frac{\partial}{x_{1}}, \frac{\partial}{x_{2}}, \frac{\partial}{x_{3}}\right)$ and the superposed dot stands for the material time derivative.

We assume the following kinematic relation for the material under study

$$
\widetilde{\varepsilon}=\widetilde{\varepsilon}^{e}+\widetilde{\varepsilon}^{p}+\tilde{p}+\tilde{\alpha}\left(T-T^{\prime}\right),
$$

where $\widetilde{\varepsilon}^{e}$ is the elastic strain tensor, $\widetilde{\varepsilon}^{p}$ is the plastic strain tensor (related to the defect motion), $\widetilde{p}$ is the defectinduced strain tensor, $\widetilde{\alpha}$ is the tensor of the thermal expansion coefficient, and $T^{\prime}$ is the reference temperature.

The free energy is assumed in the form $F\left(\widetilde{\varepsilon}^{e}, T, \tilde{p}\right)$, and thus expressions (1) become

$$
\begin{aligned}
& F_{\widetilde{\varepsilon}^{e}}: \dot{\overrightarrow{\widetilde{\varepsilon}}}^{e}+F_{T} \dot{T}+F_{\tilde{p}}: \dot{\tilde{p}}+\eta \dot{T}+\dot{\eta} T=\frac{1}{\rho} \tilde{\sigma}: \dot{\widetilde{\varepsilon}}+r-\bar{\nabla} \cdot \bar{q} \\
& -\dot{T} \eta-\bar{q} \cdot \frac{\bar{\nabla} T}{T}-F_{\widetilde{\varepsilon}^{e}}: \dot{\vec{\varepsilon}}^{e}-F_{T} \dot{T}-F_{\tilde{p}}: \dot{\tilde{p}}+\frac{1}{\rho} \tilde{\sigma}: \dot{\widetilde{\varepsilon}} \geq 0,
\end{aligned}
$$

where $F_{a}$ is the derivative of $F$ with respect to $a$. By using the kinematic relation (2), the entropy evolution expression (3.2) can now be written as

$$
-\dot{T} \eta-\bar{q} \cdot \frac{\bar{\nabla} T}{T}-F_{\widetilde{\varepsilon}^{e}}: \dot{\widetilde{\varepsilon}}^{e}-F_{T} \dot{T}+\frac{1}{\rho} \tilde{\sigma}:\left(\dot{\widetilde{\varepsilon}}^{e}+\dot{\widetilde{\varepsilon}}^{p}+\dot{\tilde{p}}+\widetilde{\beta} \dot{T}\right) \geq 0
$$

By requiring that (4) should hold for any thermodynamic process, we have

$$
\begin{aligned}
& \tilde{\sigma}=\rho F_{\varepsilon^{e}}, \\
& \eta=-F_{T}+\frac{1}{\rho} F_{\varepsilon^{e}}: \tilde{\beta}, \\
& -\vec{q} \cdot \frac{\bar{\nabla} T}{T}-F_{\tilde{p}}: \dot{\tilde{p}}+\frac{1}{\rho} \tilde{\sigma}:\left(\dot{\tilde{\varepsilon}}^{p}+\dot{\tilde{p}}\right) \geq 0 .
\end{aligned}
$$

Consequently, the energy balance (3.1) takes the form

$$
F_{\tilde{p}}: \dot{\tilde{p}}+\dot{\eta} T=\frac{1}{\rho} \tilde{\sigma}:\left(\dot{\widetilde{\varepsilon}}^{p}+\dot{\tilde{p}}\right)+r-\bar{\nabla} \cdot \vec{q}
$$

or

$$
c \dot{T}=\bar{\nabla} \bar{q}+r+Q^{e}+Q^{p}
$$


where $Q^{e}=T F_{T \varepsilon^{e}}: \dot{\widetilde{\varepsilon}}^{e}$ is the heating due to the thermoelastic effect, $Q^{p}=T F_{T p}: \dot{\vec{p}}+\frac{1}{\rho} \tilde{\sigma}: \dot{\widetilde{\varepsilon}}^{p}+\left(\frac{1}{\rho} \tilde{\sigma}-F_{p}\right): \dot{\tilde{p}}$ represents the inelastic contribution to the heating, and $c=-T F_{T T}$ is the specific heat capacity.

By using equation (6), we obtain the following expression for the stored energy rate

$$
\dot{\beta}=\frac{\left(-T F_{T \tilde{p}}+F_{\tilde{p}}\right): \dot{\tilde{p}}}{\frac{1}{\rho} \tilde{\sigma}:\left(\dot{\tilde{\widetilde{\varepsilon}}}^{p}+\dot{\tilde{p}}\right)} .
$$

Assuming $F_{T p}=0$ and $\dot{\tilde{p}}<<\dot{\widetilde{\varepsilon}}^{p}$, we can write equation (7) in more conventional form, $\dot{\beta}=F_{\tilde{p}}: \dot{\tilde{p}} / \frac{1}{\rho} \tilde{\sigma}: \dot{\tilde{\varepsilon}}$.

We assume that thermodynamic forces and fluxes are linearly related. Thus, we can obtain the following constitutive equations:

$$
\begin{aligned}
& \dot{\widetilde{\varepsilon}}^{p}=I_{\varepsilon^{p}} F_{\varepsilon^{e}}+I_{\varepsilon^{p} p}\left(F_{\varepsilon^{e}}-F_{p}\right) \\
& \dot{\tilde{p}}=I_{p}\left(F_{\varepsilon^{e}}-F_{p}\right)+I_{\varepsilon^{p} p} F_{\varepsilon^{e}},
\end{aligned}
$$

where function $F_{p}$ is given by the statistical model of a solid with mesodefects, which we describe next.

\section{Statistical model of mesodefects properties}

The parameters describing the typical mesoscopic defects (microcracks, microshears) were introduced in [18] as a localisation of the corresponding group of symmetries of the distortion tensor and can be viewed as fluctuations of the displacement field. These defects are described by symmetric tensors of the form $s_{i k}=s v_{i} v_{k}$ in the case of microcracks and $s_{i k}=1 / 2 s\left(v_{i} l_{k}+l_{i} v_{k}\right)$ for microshears. Here $v$ is unit vector normal to the base of a microcrack or slip plane of a microshear; $I$ is a unit vector in the direction of shear; $s$ is the volume of a microcrack or the shear intensity for a microshear. The microscopic kinetics for the parameter $s_{i k}$ is determined by the Langevin equation:

$$
\dot{s}_{i k}=K_{i k}\left(s_{l m}\right)-F_{i k},
$$

where $K_{i k}\left(S_{I m}\right)$ and $F_{i k}$ are, respectively, the deterministic and uncorrelated in time stochastic parts of the force field and satisfy the relations: $\left\langle F_{i k}\right\rangle=0$ and $\left\langle F_{i k}\left(t_{1}\right) F_{i k}\left(t_{2}\right)\right\rangle=Q \delta\left(t_{1}-t_{2}\right) ; \delta$ is the delta function; $Q$ is the correlation function of the fluctuating forces (nonequilibrium potential determining the energy relief of the initial structure). The size and orientation distribution function $W(s, v, I)$ of the defects is given by the Fokker-Plank equation:

$$
\frac{\partial}{\partial} W=-\frac{\partial}{\partial s_{i k}} K_{i k} W+\frac{1}{2} Q \frac{\partial^{2}}{\partial s_{i k} \partial s_{i k}} W
$$

It was shown in [18] that the Lagrangian of these mesoscopic defects can be written in the form:

$$
E=E_{0}-H_{i k} s_{i k}+\alpha s_{i k}^{2}
$$

and includes a term $H_{i k} s_{i k}$ that reflects the interaction of defects with an external field and between defects. The "effective field" $H_{i k}$ is written as a sum of an external stress field $\sigma_{i k}$ and the mean-field produced by the defects $\lambda p_{i k}$ : $H_{i k}=\gamma \sigma_{i k}+\lambda p_{i k}, p_{i k}=n\left\langle s_{i k}\right\rangle \quad$ is the macroscopic tensor of the defect density; $n$ is the defect concentration, and $\lambda$ and $\gamma$ are parameters of the material. The solution of the Fokker-Plank equation based on the assumption of statistical self-similarity of the defect distribution makes it possible to represent the distribution function in the form 
$W=Z^{-1} \exp (-E / Q)$, where $Z$ is a generalised partition function. The macroscopic value of $p_{i k}$ is determined by averaging:

$$
p_{i k}=n \int s_{i k} W(s, v, I) d s d^{3} v d^{3} I
$$

Figure 1 shows $p_{x z}$ versus $\sigma_{x z}$ for the case of a simple shear for different values of the parameter $\delta=2 \alpha / \lambda n$. Two characteristic scales determine the parameter $\delta:$ the characteristic size $I_{n}$ of the defect nucleus and the average distance $I_{c}$ between defects. The dislocation model of mesoscopic defect gives $\alpha \approx G / V_{0}$, where $G$ is the shear modulus and $V_{0}$ is the initial "free" volume (volume of the nucleus). Estimating the mean-field constant as $\lambda \approx G$ gives $\delta \approx I_{c} / I_{n}$. The solution of (12) shows that transitions to equivalent classes of curves in Fig. 1 occur when the parameter $\delta$ reached critical values $\delta_{\star}$ and $\delta_{c}$, which are bifurcation points.

It was show in [18] the curves in Fig. 1 correspond to the characteristic responses of a material to loading (quasi-brittle, ductile and nanocrystalline), and phenomenologically this behaviour can be reflected in the form of the Ginzburg-Landau expansion for the thermodynamic force $F_{p}$

$$
\frac{\partial F}{\partial \tilde{p}}=\alpha \tilde{g}+\beta \tilde{p}+\gamma \tilde{p}^{2}+\bar{\nabla} \cdot \chi \bar{\nabla} \tilde{p}
$$

where $\alpha=2 a_{1} J_{1}(\tilde{p})+3 b_{1} J_{1}^{2}(\tilde{p})+b_{2} J_{1}\left(\tilde{p}^{2}\right)+3 c_{1} J_{1}^{3}(\tilde{p})+c_{2} J_{1}\left(\tilde{p}^{3}\right)+2 c_{3} J_{1}(\tilde{p}) j_{1}\left(\tilde{p}^{2}\right)$,

$\beta=2 a_{2}+2 b_{2} J_{1}(\tilde{p})+2 c_{3} J_{1}^{2}(\tilde{p})+4 c_{4} J_{1}\left(\tilde{p}^{2}\right), \gamma=3 b_{2}+3 c_{2} J_{1}(\tilde{p})$,.

$\mathrm{J}_{1}(\tilde{p})$ is first invariant of tensor $\tilde{p}$.

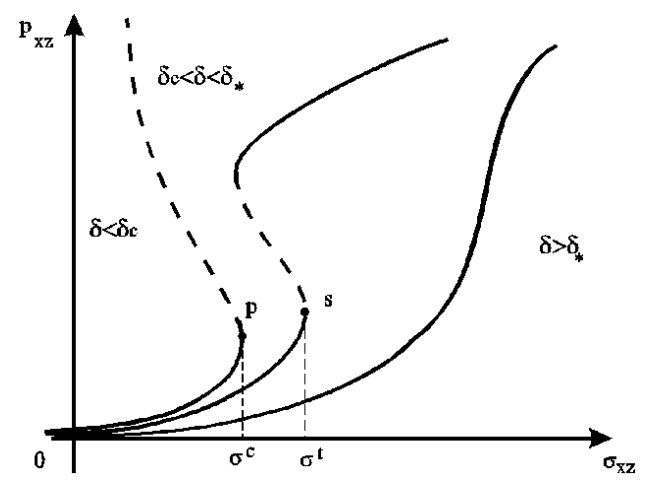

Fig.1. Characteristic responses of materials to growth of defects

Considering the "polar" character of the interaction of the defects and the appearance of nonlocality, a term quadratic in the gradient of $p_{i k}$ has been introduced in the expansion of the free energy, where $\chi$ is the nonlocality parameter.

The application of the developed model for the description of the results of infrared monitoring of quasi-static tension of metals is presented in [21,22]. The application of this model to the study of cycling loading requests some additional hypothesis. The following parts of the paper are devoted to the illustration of advantage of infrared monitoring technique and present two interesting effects accompanying fatigue loading of metals.

\section{Infrared thermography application to the study of metals fatigue}

\subsection{Material and experimental conditions}

The material under investigation is titanium Grade 2 with different grain sizes. The specimens of titanium Grade 2 were manufactured by the method of intensive plastic deformation [23] and had the grain size of about 150 nanometers. The mechanical properties of titanium Grade 2 in polycrystalline and submicrocrystalline state are presented in Table 1. The geometry of samples is represented in figure 2. The cyclic loading was carried out using a resonant electrodynamic testing machine Vibrophore Amsler providing uniaxial loading with the prescribed boundary 
conditions for stress. The resonance frequency of the sample was $76 \mathrm{~Hz}$ with the asymmetry factor of a cycle equalling 0.1 .

The experimental procedure corresponded to the express infrared techniques for determination of the fatigue limit $[8,10]$. This technique is based on the hypothesis about correlation of the value of the fatigue limit with the threshold of structural evolution. After passing the fatigue limit (loading with stress amplitude higher than the fatigue limit) an intensive structural evolution and, as a result, an intensive temperature rise was observed in the specimens. The fatigue history of each sample including "cyclic" blocks contained 30000 cycles

Table 1. Mechanical properties of titanium Grade 2 at coarse and fine grain states

\begin{tabular}{|l|c|c|c|}
\hline \multicolumn{1}{|c|}{ Type of treatment } & $\begin{array}{c}\text { tensile strength, } \sigma_{\mathrm{B}}, \\
(\mathrm{MPA})\end{array}$ & $\begin{array}{c}\text { yield stress, } \sigma_{0.2,} \\
(\mathrm{MPa})\end{array}$ & $\begin{array}{c}\text { ultimate elongation, } \\
(\delta, \%)\end{array}$ \\
\hline Initial coarse grain state (grain size $25 \mu \mathrm{m})$ & 440 & 370 & 38 \\
\hline IPD + hot rolling (grain size $0.3 \mu \mathrm{m})$ & $1090 \pm 20$ & $980 \pm 20$ & $13 \pm 1$ \\
\hline
\end{tabular}

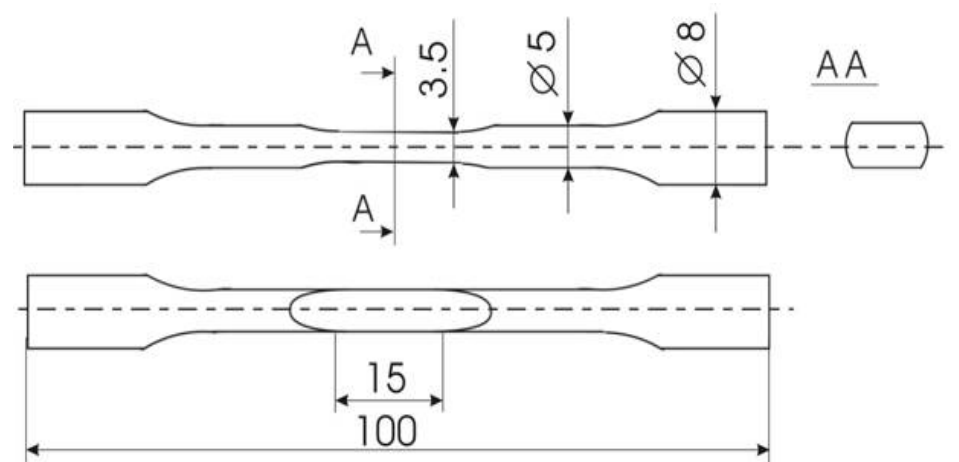

Fig. 2 Geometry of samples used for self-heating tests. The sizes of a gage part of a sample $15 \times 5 \times 3,5 \mathrm{~mm}$.

For each subsequent block the average stress was increased by $10 \mathrm{MPa}$. At each step of loading a temperature rise in the sample was measured. Between cycles the samples were unloaded and relaxed until they reached thermal equilibrium with the environment.

To study the temperature evolution at fatigue crack tip we used the plate specimen presented in figure 3 . The specimens were manufactured from a commercial purity titanium sheet $3 \mathrm{~mm}$ thick.

The surface of the specimens was polished in several stages by the abrasive paper (at the final stage of polishing the grit size does not exceed $3 \mu \mathrm{m}$ ). Before starting the experiment, the polished surface was covered by a thin layer of amorphous carbon.

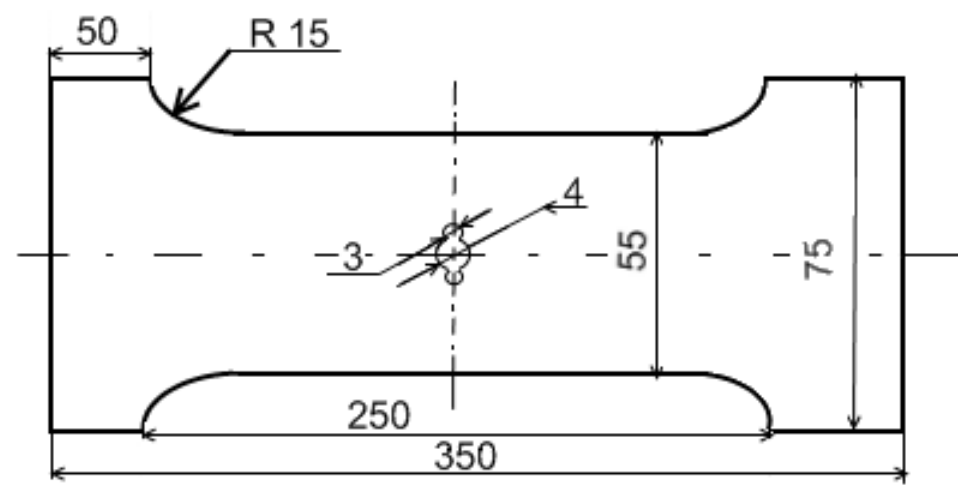

Fig. 3. Specimen geometries for investigation of temperature evolution under fatigue crack propagation.

Fatigue crack propagation tests were carried out using $100 \mathrm{kN}$ servo-hydraulic machine Bi-00-100. The strain was measured by an axial extensometer - Bi-06-304 with an accuracy of $\pm 1,5 \mathrm{~mm}$.

To study thermal effects at the crack tip, the specimen was pre-weakened by holes (Fig. 3). The fatigue crack (about $10 \mathrm{~mm}$ ) was initiated at the initial stage of the experiment by high amplitude cyclic loading of the specimens at the average stress of $215 \mathrm{MPa}$, stress amplitude of $238 \mathrm{MPa}$ and loading frequency of $20 \mathrm{~Hz}$. Then the load was decreased to slow down the rate of crack propagation, which allows us to perform detailed analysis of the heat generation processes at the crack tip. 
The temperature evolution was recorded by the infrared camera FLIR SC 5000 (thermal resolution up to $0.1 \mathrm{mK}$ and maximum framing rate up to $500 \mathrm{~Hz}$ in full frame) at the frequencies ranging from $350 \mathrm{Ho} 950 \mathrm{~Hz}$ and a minimum spatial resolution of $2 \cdot 10^{-4} \mathrm{~m}$. Calibration of the camera was made based on the standard calibration table.

\subsection{Experimental results of damage evolution study}

Figure 4 presents the results of experiments. The coarse grain titanium shows nonlinear two-step growth of heat dissipation. According to the used technique the point of crossing of straight lines in figure 4 determines the value of the fatigue limit for the coarse-grained titanium (the average stress is $80 \mathrm{MPa}$, the maximal stress is $145 \mathrm{MPa}$ ).

The results of infrared scanning of submicrocrystaline titanium show that cyclic loading is accompanied by a qualitative change in the mechanisms of dissipation. At small stress amplitudes the average temperature of the sample with a fine grain structure insignificantly exceeds the temperature of the coarse-grained titanium sample. At the stress of about the fatigue limit the picture qualitatively changes. For the stress higher than the fatigue limit of the coarse-grained titanium the increment of temperature in the submicrocrystalline sample is much less than in the samples in a polycrystalline state. A linear dependence of the temperature growth rate on the average stress was observed for all fatigue histories of submicrocrystalline samples.

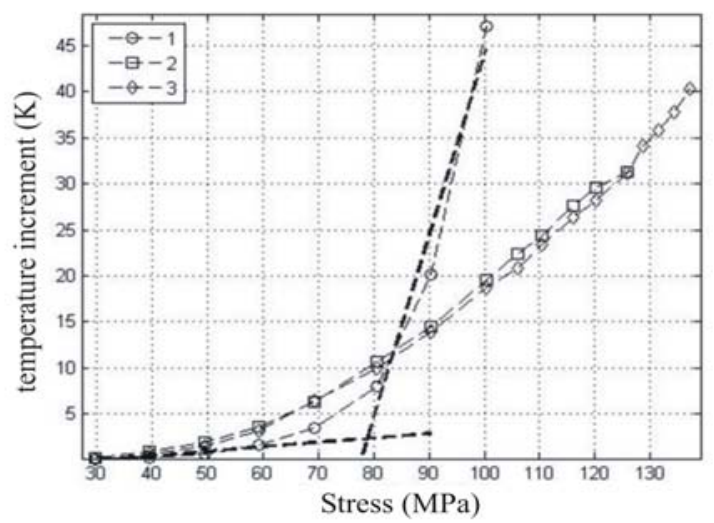

Fig. 4. Mean temperature increment of titanium samples in coarse-grained (curve 1) and submicrocrystalline states (curves 2,3) versus mean stress; dashed lines determine approximately the value of fatigue limit (the stress corresponding to the knee point).

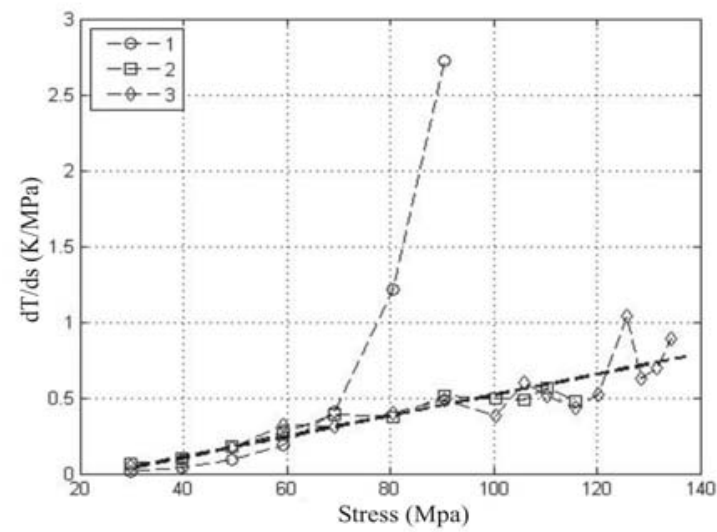

Fig. 5. The plot of mean temperature growth rate versus mean stress for coarse-grained (curve 1) and submicrocrystalline titanium (curves 2,3); dashed line denotes linear approximation of curves 2,3.

The temperature of samples is stabilized approximately after 20000 cycles which reflects the ability of the samples with submicrocrystalline structure to form an equilibrium defect system (probably grain boundary defects) and qualitatively confirms the theoretical result about the formation of defect "lattice", whose characteristic size (density of dislocation) homogeneously increases with increase in the average stress.

Submicrocrystalline samples during cyclic loading experienced brittle failure, which occurred at the stress amplitude $35 \%-40 \%$ higher than in titanium samples in an ordinary polycrystalline state. 


\subsection{Experimental results of crack propagation study}

Figure 6 shows the evolution of the maximum temperature, specific heat power and stress at the crack tip in the process of loading (220 MPa stress amplitude, mean stress of $212 \mathrm{MPa}$ and a frequency of $10 \mathrm{~Hz}$ ). The indications of the displacement sensor (don`t plotted in fig.6) mounted at different crack edges clearly demonstrate that the crack opening varies in phase with the applied stress. The analysis of the data presented in Fig.6 shows that the maximum applied stress and the maximum intensity of heat dissipation at the tip of the fatigue cracks do not coincide in time.

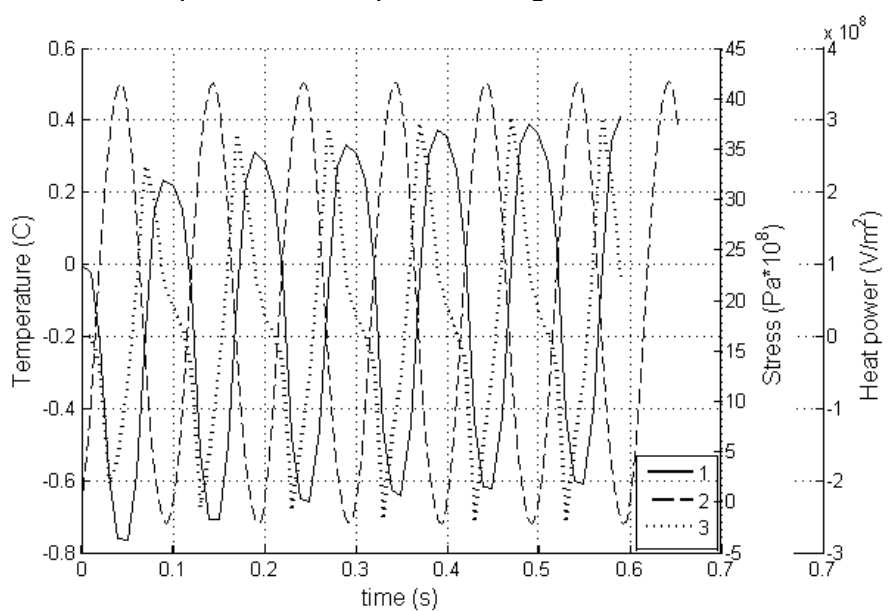

Fig. 6. Evolution of maximum temperature at the fatigue crack tip (1), stress (2), and specific heat power (3) under cyclic deformation.

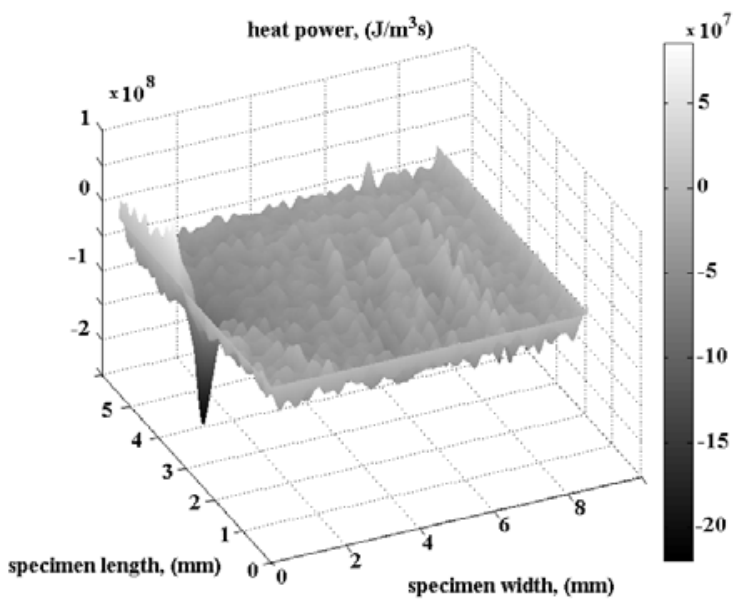

a)

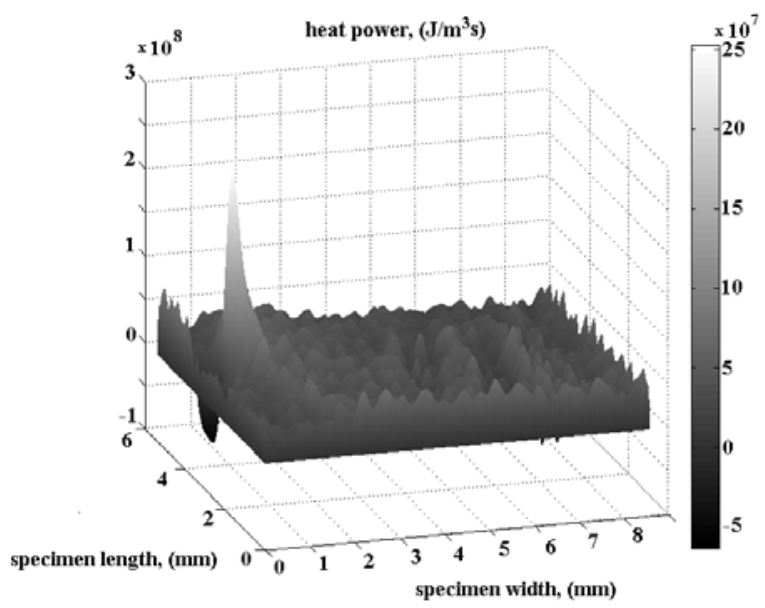

b)

Fig. 6. Specific heat power distributions on the specimen surface at different times. Thermo elastic (a) and thermoplastic (b) effects at crack tip.

To determine a specific heat power at the crack tip the corresponding algorithms including data filtration, movement compensation and a solution of inverse problem were developed. As a result a temperature field can be automatically converted in field of specific heat power. Figure 6 presents the spatial heat sources distributions on specimen surface in different time moments corresponding to the different stress levels.

To calculate the value of SIF we analyzed the low-temperature (thermoelastic) zone at the crack tip. The theoretical value of SIF can be calculated as follows:

$$
K=\sigma \sqrt{\pi \mathrm{a}} F(\alpha)
$$

where $\sigma$ is the applied stress, $a$ is half of the crack length, $\alpha=2 a / L, L$ is the specimen width, $F(\alpha)=\left(1-0.025 \alpha^{2}+0.06 \alpha^{4}\right) \sqrt{\sec (\alpha \pi / 2)}$. 


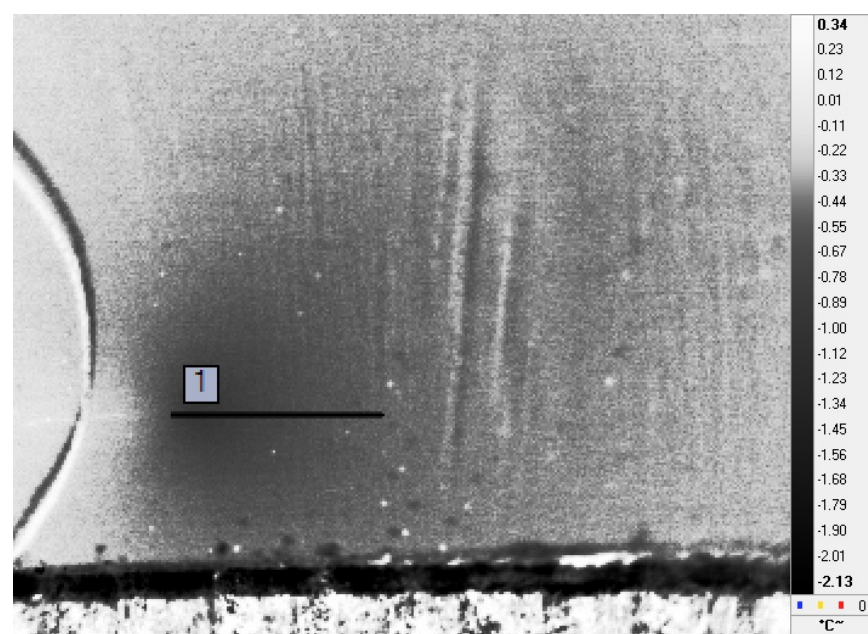

a)

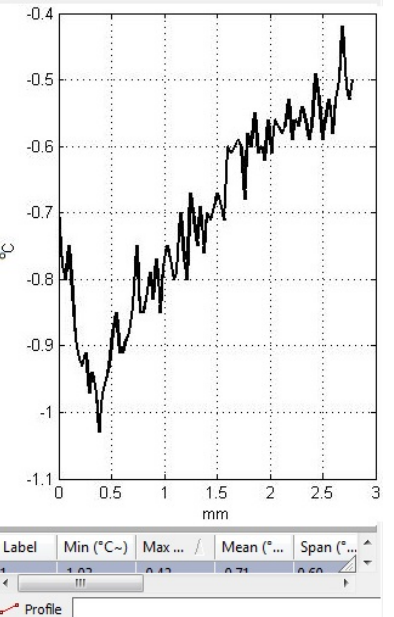

b)

Fig. 8. Temperature distribution over the specimen surface at the fatigue crack tip (a), temperature increment in the direction of crack propagation (b).

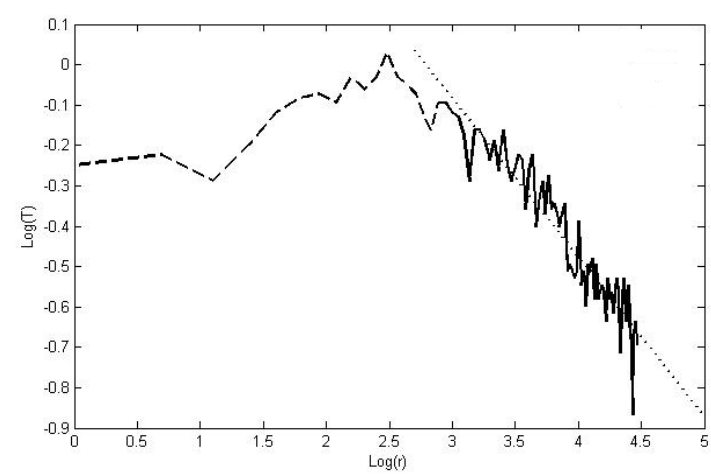

a)

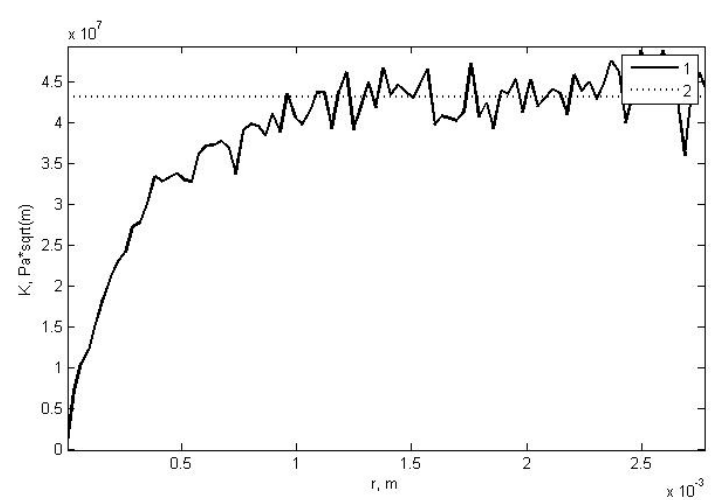

b)

Fig. 9. Temperature increment in the direction of crack propagation in log-log coordinates (a), variation of SIF with the distance from visually determined crack tip (b, horizontal line is the theoretical value of SIF).

To determine experimentally the value of SIF we can use the well known relation of thermoelasticity

$$
\Delta \sigma=-\frac{\rho C}{\alpha T^{\prime}} \Delta T
$$

where $\rho$ is the material density, $\alpha$ is the thermal expansion coefficient, $C$ is heat capacity, $\Delta T$ is experimentally determined temperature increment near the fatigue crack tip.

Let us to consider the one-dimensional function $\Delta T(r)$ determined in the direction of crack propagation (Fig 8). First, it is necessary to determine the location of the crack tip. The crack and flat specimen surface have different emissivity and crack can be easily visualized by infrared thermography. However, the existence of the cohesive force zone into the crack tip complicates the problem. This zone cannot be easily observed. Let us assume that the maximum stress corresponds to the real crack tip position. In this case, we can associate the location of minimum temperature increment with the real position of the crack tip.

Based on equation (2) we can calculate the stress increments in the direction of crack propagation $\Delta \sigma(r)=-\frac{\rho c}{\alpha T^{\prime}} \Delta T(r)$, which can be related with SIF

$$
\Delta K=\Delta \sigma(r) \sqrt{\frac{\pi r}{2}}=-\frac{\rho c}{\beta T_{0}} \Delta T(r) \sqrt{\frac{\pi r}{2}}
$$


An example of applying equation (16) for infrared monitoring data is presented in figure 8 . A slope of the last part of the plot is close to a theoretically determined value 0.5 . The first part of the plot can be considered as the result of the appearance of the cohesive force zone at the crack tip. The last part of the plot gives the constant value of SIF, which coincides with value determined by equation (14).

Figure 10 presents the comparative analysis of theoretically calculated and experimentally determined values of SIF.

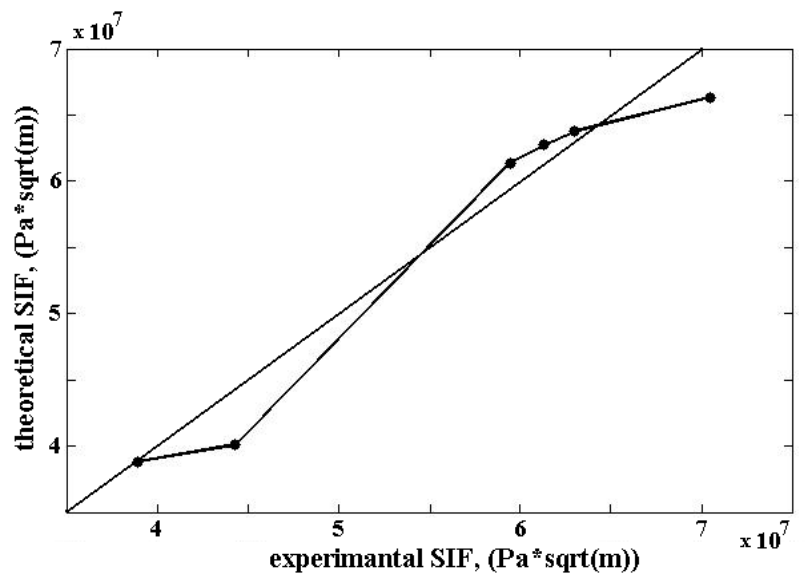

Fig. 10. Correlation of theoretically calculated and experimentally determined values of SIF.

The analysis of data presented in figure 10 shows a good agreement of experimental and theoretical results. It is necessary to note that the good agreement was obtained for relatively small stress amplitude only. The preliminary attempts (don`t presented in the framework of this paper) to calculate the value of J-integral based on the HHR-solution for stress distribution at crack tip gives significantly poorer results.

\section{Conclusion}

The present study assumes an important role of mesodefect evolution in the plastic flow of solids. The statistical consideration of the typical mesodefect ensemble coupled with the energy balance consideration allows us to determine the characteristic responses of materials to the growth of defects and to propose the constitutive equations for deformation of metals. The key point of the model is the presentation of plastic deformation in terms of two variables: plastic strain tensor (related to the defect motion) $\widetilde{\varepsilon}^{p}$ and defect induced strain tensor $\tilde{p}$. This makes it possible to consider the structural related part of plastic deformation as a thermodynamical variable and to formulate the corresponding nonequilibrium thermodynamic potential (free energy). The developed approach can be considered as a theoretical basis for investigation of energy dissipation and storage processes caused by defect evolution in metals. One of the promising experimental technique for this study is infrared thermograthy. To illustrate the advantage of this technique we carried out the experimental study of damage evolution and crack propagation under fatigue loading. In particularly, we investigated the effect of grain size on the energy dissipation process under self-heating test and applicability of linear mechanics formulae to the investigation of fatigue crack propagation.

The self-heating test shows the effect of grain size on the energy dissipation process in titanium. The polycrystalline titanium exhibits an ordinary behaviour. The temperature rises sharply after transition through fatigue limit. The temperature kinetics versus stress amplitude in submicrocrystalline titan is linear up to specimen failure. This fact allows us to conclude that submicrocrystalline titan which is characterized by specific state of ensemble of grainboundary defects has a unique structural mechanism for energy dissipation and can use this mechanism at both small and high stress amplitude.

The study of the thermoplastic effect at the fatigue crack tip has shown that the process of heat dissipation is essentially nonlinear. At this stage of research the results of experimental study suggest that the maximum heat is reached on the descending branch of the load.

The preliminary attempts to calculate the value of $\mathrm{J}$-integral based on the HHR-solution for stress distribution at crack tip gives unsatisfactory results. The good precision in the calculation of SIF can be considered based on the thermoelasticity theory for relatively small stress amplitude.

\section{Acknowledgement}

The author sincerely thanks the laboratory LAMEFIP ENSAM (Bordeaux, France) for providing of experimental equipment for some of the experiments and $\mathrm{Dr}$ T. Palin-Luc and Dr. N. Saintier for useful discussing. The work is partially supported by grants RFBR (11-01-00153, 11-01-96005) and grant МД-2684.2012.1. 


\section{REFERENCES}

[1] Oliferuk W., Maj M., Raniecki B. "Experimental analysis of energy storage rate components during tensile deformation of polycrystals". Materials Science and Engineering A, v. 374, pp.77-81, 2004.

[2] Dillon. O.W. "Coupled thermoplasticity". J. Mech. Phys. Solid., №11, pp. 21-33, 1963.

[3] Lee E.H. "Elastic plastic deformation at finite strains", J. Appl. Mech., №36, pp. 1-6, 1969.

[4] Mroz Z., Raniecki B. "On the uniqueness problem in coupled thermoplasticity", Int. J. Eng. Sci., №14, pp.211221, 1976.

[5] Simo J.C., Miene C. "Associative coupled thermoplasticity at finite strains: Formulation, numerical analysis and implementation”. Computer Methods in Applied Mechanics and Engineering, №98, pp.41-104, 1992.

[6] Bever M.B., Holt D.L., Tichener A.L. "The stored energy of cold work”, Prog. Mat. Sci., №17, pp.1-190, 1973.

[7] Farren W.S., Taylor G.I. "The heat developed during plastic extension of metals", Proc. Roy. Soc. London., A107, pp.422-451, 1925.

[8] La Rosa G., Risitano A. "Thermographic methodology for rapid determination of the fatigue limit of materials and mechanical components". International Journal of Fatigue, №22, pp. 65-73, 2000.

[9] Kapoor R., Nemat-Nasser S. "Determination of temperature rise during high strain rate deformation". Mechanics of materials, №27, pp.1-12, 1998.

[10] Luong M.P. "Infrared thermographics scanning of fatigue in metals". Nuclear Engineering and Design, №158, pp. ${ }^{\circ} 363-376,1995$.

[11] Plekhov O., Palin-Luc T., Naimark O., Uvarov S., Saintier N. "Fatigue crack initiation and growth in a 35CrMo4 steel investigated by infrared thermography", Fatigue and fracture of engineering materials and structures, Volume 28, Issue 1, pp 169-178, 2005.

[12] Chrysochoos A., Louche H. "An infrared image processing to analyse the calorific affects accompanying strain localization”. International journal of engineering science, №38, pp.1759-1788, 2001.

[13] Paynter R.J.H., Dutton A.G. "The use of a second harmonic correlation to defect damage in composite structures using thermoelastic stress measurements", Strain, №39, pp.73-78, 2003.

[14] Andrianopoulos N.P., Champidis K.T., lliopoulos A.P. "Detection of crack nucleation in sheet metal forming by monitoring infrared radiation”, Fatigue Fract Engng Mater Sruct, №26, pp. 323-328, 2003.

[15] Wolfenden A. Appleton A.S. "The energy stored during the low-temperature deformation of copper and aluminum single crystals", Acta met., №16, issue 7, pp. 915-925, 1968.

[16] Mason J.J., Rosakis A.J., Ravichandran G. "On the strain rate dependence of the fraction of plastic work converted to heat: an experimental study using speed infrared detectors and Kolsky bar". Mechanics of materials, №17, pp. 135-145, 1994.

[17] Rosakis P., Rosakis A.J., Ravichandran G., Hodowany J. "A thermodynamic internal variable model for the partition of plastic work into heat and stored energy in metals". J. Mech. and Phys. Solids., №48, pp.581-607, 2000.

[18] Naimark O.B. "Kinetic transition in ensembles of microcrack and some nonlinear aspects of fracture", IUTAM Symposium on Nonlinear Analysis of Fracture, Cambridge, 285, 1997.

[19] Plekhov O., Naimark O., Valiev R., Semenova I. "Investigation of energy balance in nanocrystalline titanium under cyclic loading". Damage and Fracture Mechanics, Boukharouba, Taoufik; Elboujdaini, Mimoun; Pluvinage, Guy (Eds.). Springer Netherlands. pp. 386-391, 2009.

[20] Landau L.D., Lifshitz E.M. "Course of Theoretical Physics. Statistical Physics”. Pergamon Press, Oxford, 1980.

[21] Plekhov. O., Naimark O., "Theoretical and experimental study of energy dissipation in the course of strain localization in iron". Journal of Applied Mechanics and Technical Physics, Vol. 50, No. 1, pp. 1-18, 2009.

[22] Plekhov O. "Experimental Study of Thermodynamics of Plastic Deformation by Infrared Thermography". Technical Physics, Vol. 56 No. 2, pp. 301-304, 2011.

[23] Latysh V., Semenova I., Salimgareeva G., Kandarov I., Zhu Y., Lowe T., Valiev R. Mater. Sci. Forum, Vol. 503504, pp. 763-769, 2006. 